\title{
Comprehensive longitudinal study of epigenetic mutations in aging
}

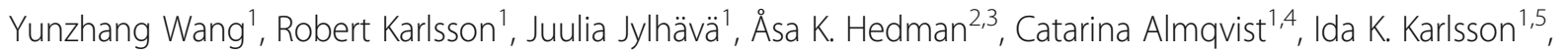
Nancy L. Pedersen", Malin Almgren ${ }^{6}$ and Sara Hägg ${ }^{1 *}$

\begin{abstract}
Background: The role of DNA methylation in aging has been widely studied. However, epigenetic mutations, here defined as aberrant methylation levels compared to the distribution in a population, are less understood. Hence, we investigated longitudinal accumulation of epigenetic mutations, using 994 blood samples collected at up to five time points from 375 individuals in old ages.

Results: We verified earlier cross-sectional evidence on the increase of epigenetic mutations with age, and identified important contributing factors including sex, CD19+ B cells, genetic background, cancer diagnosis, and technical artifacts. We further classified epigenetic mutations into High/Low Methylation Outliers (HMO/LMO) according to their changes in methylation, and specifically studied methylation sites (CpGs) that were prone to mutate (frequently mutated CpGs). We validated four epigenetically mutated CpGs using pyrosequencing in 93 samples. Furthermore, by using twins, we concluded that the age-related accumulation of epigenetic mutations was not related to genetic factors, hence driven by stochastic or environmental effects.
\end{abstract}

Conclusions: Here we conducted a comprehensive study of epigenetic mutation and highlighted its important role in aging process and cancer development.

Keywords: Epigenetic mutation, Aging, Cancer, Twin study

\section{Introduction}

Epigenetic processes, among which DNA methylation is one of the most well studied, are fundamental in human aging [1]. Studies on DNA methylation have identified age-associated changes in methylation levels shared by individuals [2, 3], and have also reported an increasing divergence of methylation levels between individuals with age $[4,5]$.

Epigenetic mutations, defined as aberrant methylation levels that can lead to unusual gene expression, may be involved in cancer development and important for human aging [6, 7]. Unlike age-associated changes in methylation levels that are shared among individuals, the incidences of epigenetic mutations are rare, stochastic, and inconsistent between individuals. Recently, emerging studies on methylation variability have also identified differentially varied CpGs associated with cancer field

\footnotetext{
* Correspondence: sara.hagg@ki.se

${ }^{1}$ Department of Medical Epidemiology and Biostatistics, Karolinska Institutet, Nobels väg 12A, 17177 Stockholm, Sweden

Full list of author information is available at the end of the article
}

defects $[8,9]$. Epigenetic mutations can partly explain the increasing variability of methylation levels between individuals over time, but conversely, highly varied methylation sites do not necessarily contain extreme outliers. The extreme methylation levels may concur stronger biological consequences, such as cancer. Epigenetic mutations could contribute to the aging process through the accumulation of abnormally methylated CpGs (cytosine-phosphatase-guanine sites), which could further cause abnormal gene expression and downstream effects in tissues. A previous study by Gentilini et al [7] specifically defined epigenetic mutations as extreme outliers within a population, with methylation levels exceeding three times interquartile ranges (IQR) of the first quartile $(\mathrm{Q} 1-3 \times \mathrm{IQR})$ or the third quartile $(\mathrm{Q} 3+3 \times \mathrm{IQR})$. They found that the total numbers of epigenetic mutations increased exponentially with age. Also, studies using a similar outlier definition have identified methylation outlier in association with undesirable birth outcomes [10] and cancer [11]. However, the study on epigenetic mutations and aging was based on a cross- 
sectional study, it needs to be validated in a longitudinal setting, where the accumulation of epigenetic mutations over time can be followed within the same individuals. Moreover, it is not yet known what the clinical consequences of accumulated epigenetic mutations are, and if individuals with a high burden of epigenetic mutations are prone to develop cancer as previously suggested $[6,12]$.

In this study, we used a Swedish twin cohort including 375 individuals sampled up to five times in late life across 18 years (Table 1). We first validated the age-related increase of epigenetic mutations from a longitudinal perspective. Next, we identified important factors associated with the number of epigenetic mutations, including sex, cellular composition (CD19 B cells), genetic background, and technical artifacts. In parallel, we analyzed the direction of change in methylation level and characterized the epigenetic mutations as High- (HMO) and Low Methylation Outliers (LMO). We also studied the association between epigenetic mutations and cancer, as well as the genetic influence on epigenetic mutations using a twin approach. Last, we validated a select set of epigenetic mutations using bisulfite pyrosequencing.

\section{Results}

\section{Longitudinal accumulation of epigenetic mutations is} exponentially associated with age

To explore the longitudinal increase in number of epigenetic mutations, we measured DNA methylation data (Illumina 450k array) repeatedly in whole blood samples $(n=994)$ from participants in the Swedish Adoption/ Twin Study of Aging (SATSA; Table 1) [13]. To avoid confounding by underlying genetic variation, we removed 20,660 CpGs that were associated with at least one single nucleotide polymorphism (SNP) $(p<1 \mathrm{e}-14)$ within $1 \mathrm{Mbps}$ (mega base pairs), i.e., cis-methylation quantitative loci (cis-meQTLs). In the remaining 370, $234 \mathrm{CpGs}$, the number of epigenetic mutations ranged from 58 to 26,291 in each sample, using the definition in Gentilini et al [7]. Across samples, the number of epigenetic mutations had a right-skewed distribution, which was close to normal distribution after $\log 10$ transformation (Additional file 1: Figure S1).

After identifying epigenetic mutations in SATSA, we found that the $\log 10$ total number of epigenetic mutations increased with age ( $p=1.22 \mathrm{e}-13)$ longitudinally (Fig. 1a). We also identified additional factors and confounders associated with the number of epigenetic mutations (Table 2). Women had a slightly higher average number of epigenetic mutations than men $(p=$ 6.33e-3). Low sample quality, as defined by the $\log 10$ transformed number of CpGs with detection $p$ values over 0.01 , was positively associated with the total number of epigenetic mutations $(p=1.48 \mathrm{e}-117)$. In general, unreliable samples tended to have more epigenetic mutations, indicating that measurement errors could also be identified as epigenetic mutations. However, after adjusting the mixed models for detection $p$ value, the effect of age on number of epigenetic mutations remained unchanged. Using predicted cellular compositions, CD19+ B cell composition was positively associated with the total number of epigenetic mutations $(p=5.06 \mathrm{e}-23)$. After removing cis-meQTLs, the first genetic principal component (PC) showed only a minor effect on the total number of epigenetic mutation $(p=0.041)$.

Out of all CpGs, 237,398 (64\%) were defined as epigenetic mutations in at least one sample, but only 1,185 (0.32\%) CpGs were mutated in more than 50 samples (5\% samples), subsequently defined as frequently mutated CpGs. Only two of the 1,185 frequently mutated CpGs were also identified to be age-differentially methylated sites (aDMS) in our previous study [3]. The frequently mutated CpGs were still significantly associated with age, sample quality, CD19+ B cell compositions, and genetic PC1, while sex was no longer significant (Table 2).

\section{High/low methylation outliers}

Compared to normal methylation levels in the population, epigenetic mutations can be either higher or lower in methylation level. Hence, we defined HMO and LMO as CpGs with abnormally higher or lower methylation levels than the average (Additional file 1: Figure S2). Of the defined epigenetic mutation sites, almost half were identified as HMOs and the other half as LMOs (118, 259 HMOs and 119,175 LMOs). Thirty-six CpGs were defined as both HMOs and LMOs because those sites had intermediate methylation levels and very small IQRs. However, among the frequently mutated CpGs, there

Table 1 Characteristics of study participants in SATSA ( $n=375$ unique individuals)

\begin{tabular}{lllll}
\hline Longitudinal wave & Year of sample collection & Number of participants (new recruits) & Female proportion & Age mean (SD) \\
\hline 1 & $1992-1994$ & 232 & $58 \%$ & $68.5(9.1)$ \\
2 & $1999-2001$ & $239(101)$ & $63 \%$ & $71.1(10.1)$ \\
3 & $2002-2004$ & $186(25)$ & $54 \%$ & $72.1(9.1)$ \\
4 & $2008-2010$ & $183(14)$ & $61 \%$ & $76.2(8.5)$ \\
5 & $2010-2012$ & $154(3)$ & $66 \%$ & $77.0(8.4)$ \\
\hline
\end{tabular}



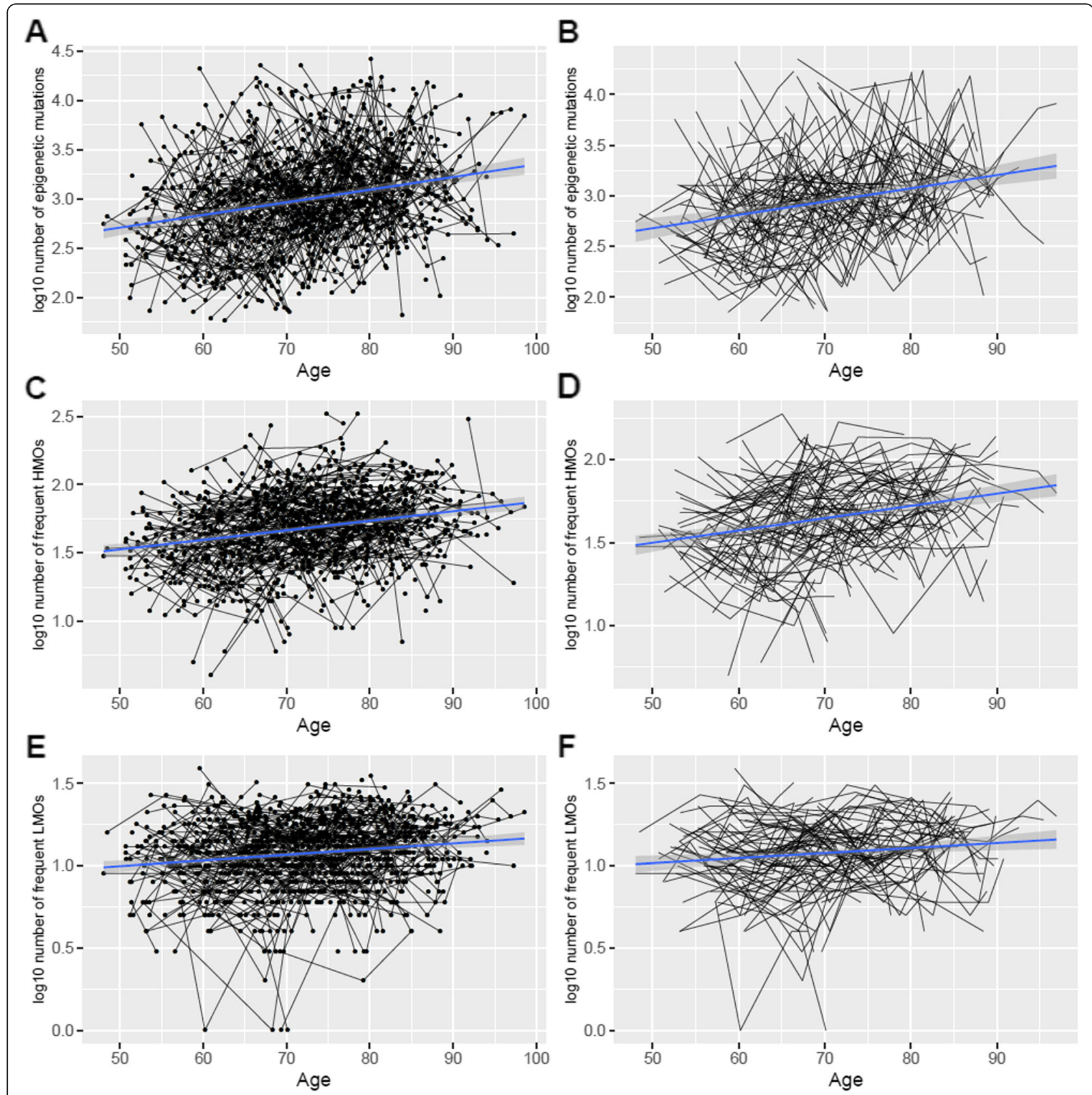

Fig. 1 The number of epigenetic mutations (log10-transformed) increased longitudinally with age in a longitudinal perspective using genomewide DNA methylation data from repeated whole blood samples collected in the Swedish Adoption/Twin Study of Aging (SATSA; $n=375$ participants). The numbers of epigenetic mutations of samples were counted from a total epigenetic mutations ( $n=370,234 \mathrm{CpGs}, p=1.22 \mathrm{e}-13$ for association with age), $\mathbf{b}$ total epigenetic mutation in individuals with at least 4 measures ( $p=1.94 \mathrm{e}-08$ for association with age), $\mathbf{c}$ frequent high methylation outliers (HMOs) ( $n=969 \mathrm{CpGs}, p=2.09 \mathrm{e}-17$ for association with age), $\mathbf{d}$ frequent HMOs in individuals with at least 4 measures ( $p=1.07$ e-10 for association with age), e frequent low methylation outliers (LMOs) $(n=216 \mathrm{CpGs}, p=1.14 \mathrm{e}-05$ for association with age), and f frequent LMOs in individuals with at least 4 measures ( $p=5.76 \mathrm{e}-03$ for association with age)

were significantly more HMOs than LMOs (969 and 216, $p<1 \mathrm{e}-16$ ) (Fig. 2). Similar to the results of epigenetic mutations, both HMOs and LMOs were significantly associated with age $(p=1.98 \mathrm{e}-12$ for HMOs and $p=$ $1.73 \mathrm{e}-14$ for LMOs), sex ( $p=1.81 \mathrm{e}-3$ for HMOs and $p=$ 0.037 for LMOs), and B cells $(p=3.76 \mathrm{e}-22$ for HMOs and $p=3.03 \mathrm{e}-20$ for LMOs). Nevertheless, numbers of both sets of frequent mutations (log10-transformed) significantly increased with age ( $p=2.09 \mathrm{e}-17$ for HMOs and $p=1.14 \mathrm{e}-05$ for LMOs) (Fig. 1c, e). Sex was no longer a significant factor with either frequent HMOs or LMOs. The composition of CD19+ B cell was still 
Table 2 The association between number of epigenetic mutations (log10-transformed) and age from mixed models with confounders

\begin{tabular}{lllllll}
\hline Number of epigenetic mutations & \multicolumn{1}{l}{ Effect sizes $(p$ values) } & & & \\
\cline { 2 - 7 } & Age (year) & $\begin{array}{l}\text { Sex } \\
\text { (Female to male) }\end{array}$ & $\begin{array}{l}\text { CD19+ B cells } \\
\text { (proportion) }\end{array}$ & $\begin{array}{l}\text { 1st genetic principal } \\
\text { component }\end{array}$ & $\begin{array}{l}\text { Sample } \\
\text { quality* }\end{array}$ & $\begin{array}{l}\text { Cancer } \\
\text { diagnosis }\end{array}$ \\
\hline Total epigenetic mutations & $8.29 \mathrm{e}-03(1.22 \mathrm{e}-13)$ & $0.0722(6.33 \mathrm{e}-03)$ & $4.21(5.06 \mathrm{e}-23)$ & $0.445(0.0413)$ & $0.369(1.48 \mathrm{e}-117)$ & $0.0697(0.0139)$ \\
Frequent epigenetic mutations & $6.03 \mathrm{e}-03(2.17 \mathrm{e}-19)$ & $-0.0180(0.33)$ & $1.76(1.37 \mathrm{e}-12)$ & $0.595(1.28 \mathrm{e}-04)$ & $0.0573(5.84 \mathrm{e}-13)$ & $0.0478(0.0164)$ \\
Frequent high methylation outliers & $6.81 \mathrm{e}-03(2.09 \mathrm{e}-17)$ & $-0.0314(0.16)$ & $2.09(2.25 \mathrm{e}-12)$ & $0.750(7.65 \mathrm{e}-05)$ & $0.0512(3.58 \mathrm{e}-08)$ & $0.0602(0.0130)$ \\
Frequent low methylation outliers & $2.82 \mathrm{e}-03(1.14 \mathrm{e}-05)$ & $0.0340(0.057)$ & $0.474(0.046)$ & $0.0186(0.92)$ & $0.0888(8.09 \mathrm{e}-30)$ & $-6.99 \mathrm{e}-03(0.71)$ \\
\hline
\end{tabular}

*Sample quality was indicated by the log10-transfromed number of CpGs with a detection $p$ value over 0.01

strongly associated with HMOs $(p=2.25 \mathrm{e}-12)$, but only marginally significant for LMOs $(p=0.046)$. Sample quality, as measured by detection $p$ value, showed strong effects on both frequent HMOs and LMOs; however, LMOs were much more influenced $(p=8.09 \mathrm{e}-30)$ than HMOs $(p=3.58 \mathrm{e}-8)$. Moreover, the first genetic principal component became a significant factor $(p=7.65 \mathrm{e}-5)$ when analyzing frequent HMOs, while it had no effect on LMOs $(p=0.92)$ (Table 2).

To better present the longitudinal effect, the same analysis was performed on 110 individuals with four or more measures (in total 470 samples). Still, the total epigenetic mutations, frequent mutations, frequent HMOs, and LMOs all significantly increased with age (Fig. 1b, d, f) despite the lower statistical power (Additional file 1: Table S1). Among the other factors, sample quality and CD19 B cell proportion were still significantly associated with all the four outcomes, while the effects of sex and genetic $\mathrm{PC} 1$ were no longer significant (Additional file 1: Table S1).

\section{Functional annotation of epigenetic mutations}

To characterize HMO and LMOs, we examined their locations in relation to $\mathrm{CpG}$ island regions and regulatory features. Compared to all CpGs analyzed, where 33.5\% of CpGs located in CpG islands, HMOs were enriched within CpG islands (63\% of CpGs, $p<1 \mathrm{e}-16)$ and frequent HMOs even more so ( $88 \%$ of CpGs, $p<1 \mathrm{e}-16$ ). On the other hand, LMOs were mostly located outside of CpG islands (88\% CpGs outside of CpG islands, $p<$ 1e-16), but the opposite was true for frequent LMOs, which were enriched in CpG islands (51\% of CpGs, $p=$ 8.6e-8) (Fig. 3). We further explored regulatory features of the frequent epigenetic mutations using the Ensembl

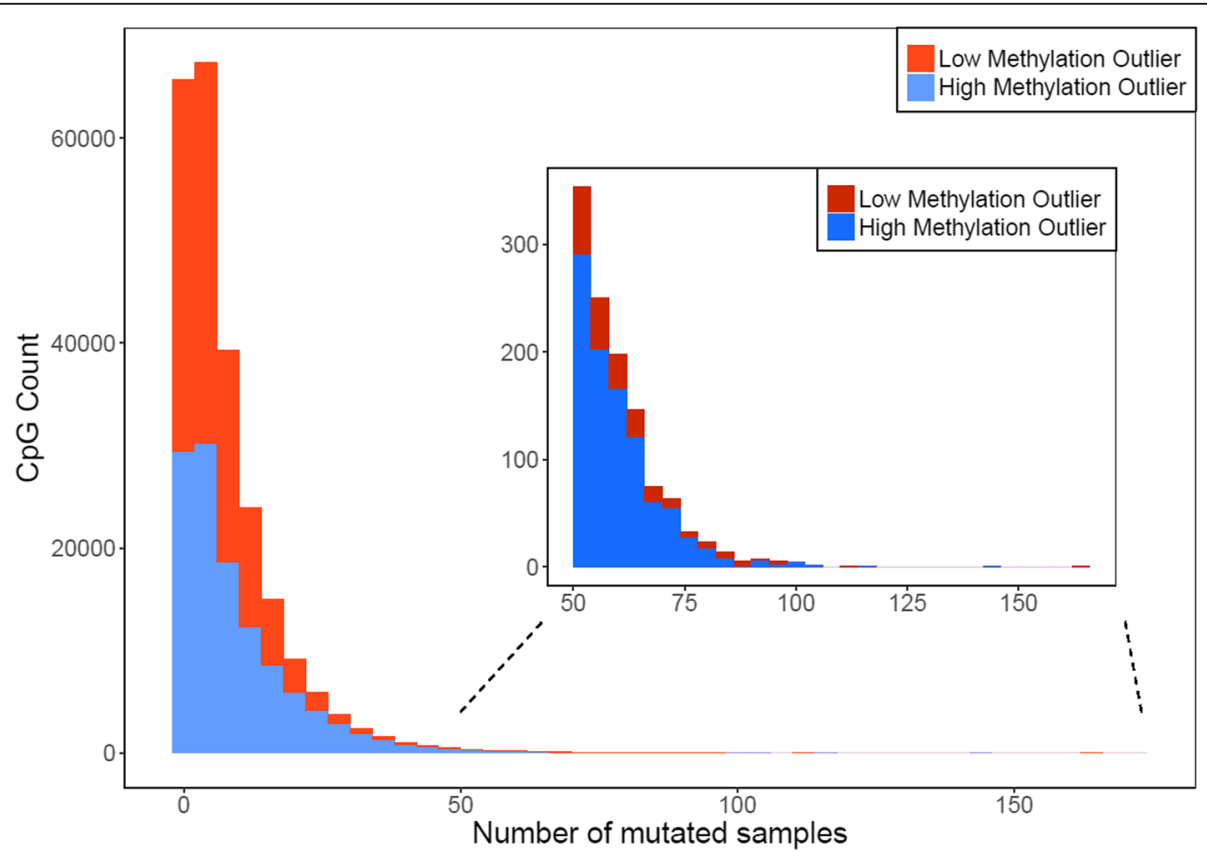

Fig. 2 The distribution of mutated samples for high methylation outliers (HMOs) and low methylation outliers (LMOs). For most CpGs, epigenetic mutations only occurred in a small number of samples, but HMOs were more likely to appear in a large number of samples $(n>50)$ than LMOs (969 HMOs and 216 LMOs, $p<1$ e-16) 


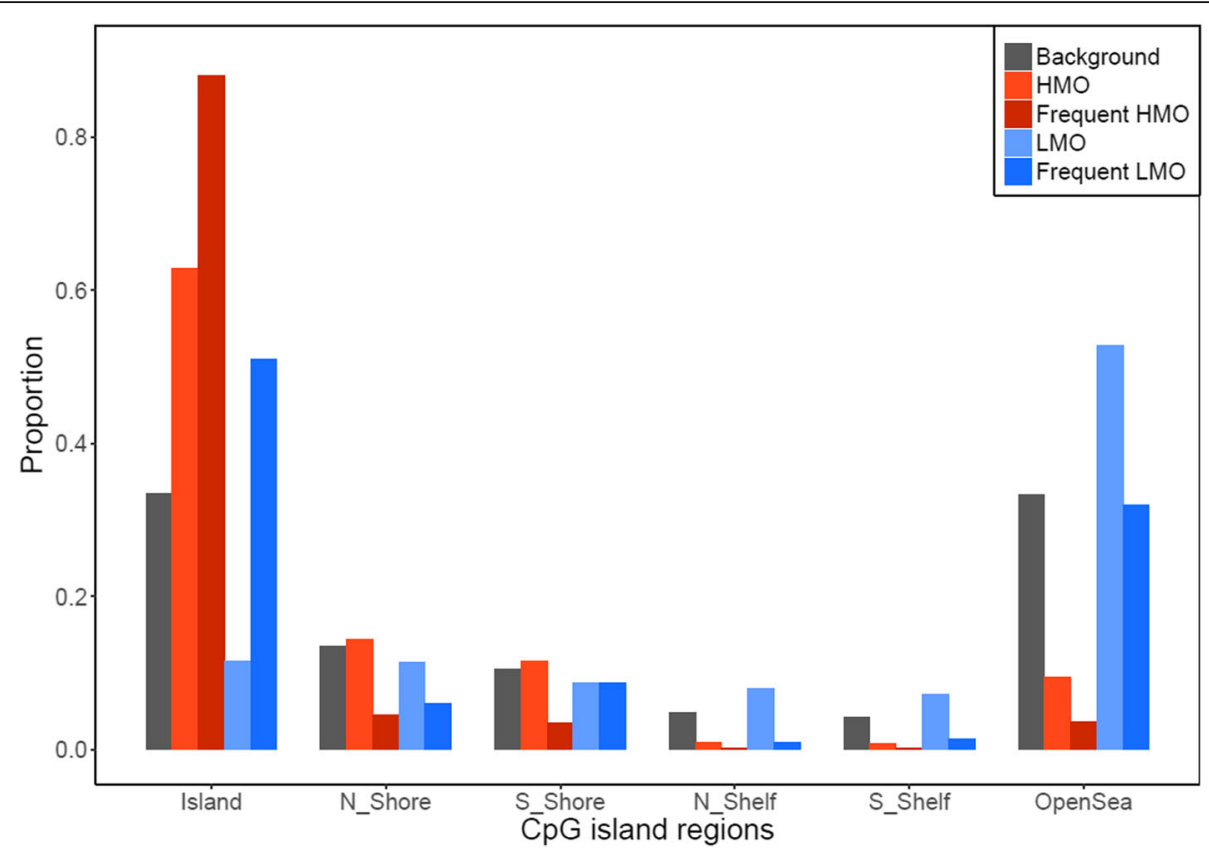

Fig. 3 Proportions of high methylation outliers (HMOs) and low methylation outliers (LMOs) in different CpG island regions. HMOs are enriched in CPG islands $(p<1 \mathrm{e}-16)$ while LMOs are more distributed outside of CpG islands ( $p<1 \mathrm{e}-16)$, especially in open sea regions. However, both frequent HMOs and LMOs are enriched in CpG islands ( $p<1 \mathrm{e}-16$ and $p=8.6 \mathrm{e}-8)$

database [14], and found that frequent HMOs were enriched in promoter regions $(p=1.1 \mathrm{e}-10)$, but less likely to be found in CCCTC-Binding factor (CTCF) binding sites $(p=1.4 \mathrm{e}-09)$ and regions of open chromatin $(p=3.6 \mathrm{e}-07)$ (Fig. 4a). The frequent LMOs, on the other hand, were enriched in CTCF $(p=7.7 \mathrm{e}-12)$ and transcription factor binding sites $(p=3.9 \mathrm{e}-05)$, open chromatin $(p=0.0012)$, and promoter flanking regions $(p=0.041)$, while depleted in promoter regions $(p=$ 6.9e-19) (Fig. 4b). Moreover, we performed a pathway analysis of frequent epigenetic mutations using DAVID [15], but failed to identify enriched pathway.

\section{Epigenetic mutation is associated with cancer diagnosis}

As aberrant DNA methylation levels in gene regulatory regions may cause abnormal gene expression, which may be associated with cancer, we analyzed epigenetic mutations in relation to cancer diagnosis in the SATSA participants. Cancer diagnosis date was retrieved using linkage to The National Patient Registry (prior to May 2016) including ICD-codes for all cancer types (ICD7 codes 140-205, ICD8 codes 140-209, ICD9 codes 140208, ICD10 codes C00-C97, and B21). The SATSA participants included 29 prevalent cancer cases diagnosed already at baseline, and 79 incident cases that developed cancer during the follow-up period. Hence, information on whether the participant was diagnosed with cancer by the end of the follow-up was tested in the mixed model for associations with log10-transformed numbers of epigenetic mutations. Samples of individuals with cancer, including samples before and after cancer diagnosis, were observed to have a significantly higher number of epigenetic mutations $(p=0.014)$, HMOs $(p=0.019)$, LMOs $(p=0.027)$, and frequent HMOs $(p=0.013)$, but no associations were found for frequent LMOs $(p=$ 0.71 , Table 2). Furthermore, in the survival analysis, people with a higher number of frequent HMOs had a higher risk of cancer incidence (Additional file 1: Table S2).

\section{Epigenetic mutations are shared within twin pairs}

By applying a co-twin control design, we could further study the genetic effect and the genetic-age interaction in association with epigenetic mutations. We calculated the number of shared epigenetic mutations within a twin pair sampled at the same time, and studied their association with time and twin zygosity using a random effects model (Table 3). The numbers of shared epigenetic mutations were normalized in order to compare the effect sizes from different sets of CpGs. First, taking all CpGs into account $(n=390,894)$, the number of shared epigenetic mutations increased significantly with age $(\beta=$ $0.019, p=0.026)$, and MZ pairs shared more epigenetic mutations than DZ pairs $(\beta=1.078, p=3.41 \mathrm{e}-18)$. After excluding 20,660 cis-meQTL CpGs, the age effect became stronger $(\beta=0.025, p=5.98 \mathrm{e}-3)$ while the zygosity effect was smaller $(\beta=0.855, p=1.05 \mathrm{e}-11)$. Last, within the 20,660 cis-meQTL-CpGs, the number of shared 

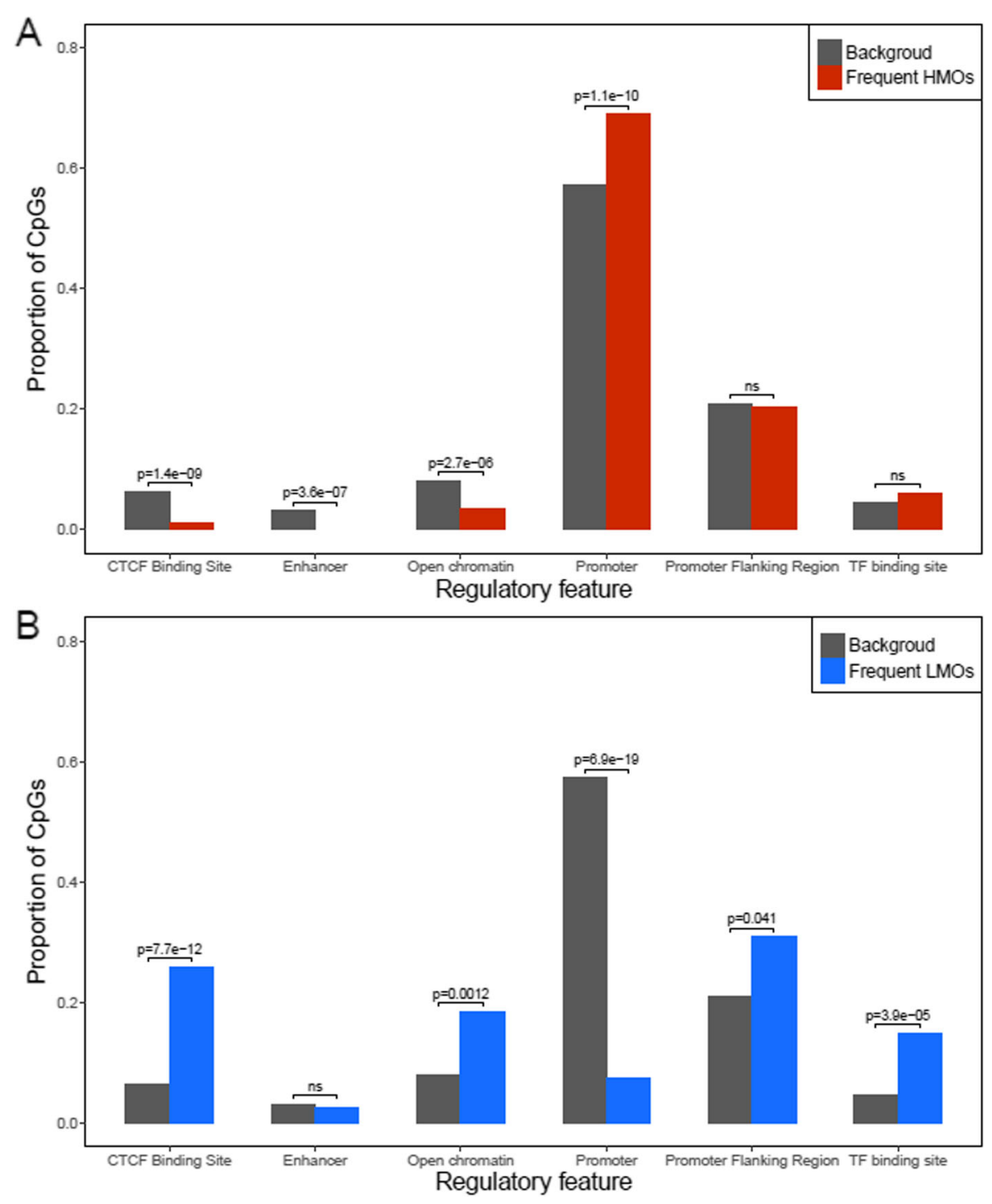

Fig. 4 The distribution of regulatory features of frequent high methylation outliers (HMOs) and low methylation outliers (LMOs). Compared to the background distribution of the 450k array design, frequent HMOs were enriched in promoter regions (a), while the opposite was true for LMOs (b)

epigenetic mutations was not associated with age $(\beta=$ $2.86 \mathrm{e}-4, p=0.969)$, while the zygosity difference $(\beta=$ $1.461, p=8.34 \mathrm{e}-28$ ) was larger than in results from nonmeQTL-CpGs. None of the three tests showed significant twin zygosity-age interaction or sex effect.

\section{Epigenetic mutations were validated using pyrosequencing}

To verify epigenetic mutations identified from $450 \mathrm{k}$ array, we selected four frequently mutated CpGs (One HMO: cg05270750 and three LMOs: cg17338133, cg25351353, cg05124918) in 93 samples from 26 individuals for validation with pyrosequencing. In general, the pyrosequencing results were well correlated with methylation data measured by the $450 \mathrm{k}$ array (cg05270750: $r=0.84$; cg17338133: $r=0.59$; cg25351353: $r=0.80$; $\operatorname{cg} 05124918: r$ $=0.77$ ). In addition, we compared methylation levels of mutated samples to the normal group using results from the $450 \mathrm{k}$ array and pyrosequencing respectively. In pyrosequencing data, significant differences were observed between mutated samples and normal ones, using the same definition of a mutated sample as that for the 450k array data (Table 4). Hence, pyrosequencing technically validated epigenetic mutations identified from the $450 \mathrm{k}$ array. Although the agreement between the two methods was generally good, we still observed large differences between pyrosequencing and $450 \mathrm{k}$ data in some samples, where four samples in cg17338133 and six samples in cg 05124918 
Table 3 The results of the scaled number of shared epigenetic mutations calculated from different sets of CpGs in association with age, sex, twin zygosity, and zygosity-age interaction

\begin{tabular}{|c|c|c|c|c|}
\hline & Covariates & Estimate & Standard error & $p$ value \\
\hline \multirow[t]{4}{*}{ All CpGs $(390,894)$} & Age & 0.019 & $8.59 \mathrm{e}-3$ & 0.026 \\
\hline & Sex & 0.208 & 0.107 & 0.055 \\
\hline & Zygosity (DZ) & -1.078 & 0.105 & $3.41 \mathrm{e}-18$ \\
\hline & Zygosity $(\mathrm{DZ}) \times$ age & -0.012 & 0.011 & 0.284 \\
\hline \multirow[t]{4}{*}{ Non-cis-meQTL CpGs $(370,234)$} & Age & 0.025 & $9.17 e-3$ & $5.98 \mathrm{e}-03$ \\
\hline & Sex & 0.183 & 0.116 & 0.117 \\
\hline & Zygosity (DZ) & -0.855 & 0.114 & $1.05 \mathrm{e}-11$ \\
\hline & Zygosity $(\mathrm{DZ}) \times$ age & -0.013 & 0.012 & 0.263 \\
\hline \multirow[t]{4}{*}{ Cis-meQTL CpGs $(20,660)$} & Age & $2.86 \mathrm{e}-4$ & $7.61 e-3$ & 0.969 \\
\hline & Sex & 0.194 & 0.107 & 0.071 \\
\hline & Zygosity (DZ) & -1.461 & 1.105 & $8.34 \mathrm{e}-28$ \\
\hline & Zygosity $(\mathrm{DZ}) \times$ age & $-3.77 e-3$ & $9.64 \mathrm{e}-3$ & 0.696 \\
\hline
\end{tabular}

meQTL: methylation quantitative trait loci

showed over $15 \%$ methylation level differences between 450k array and pyrosequencing data after centering their mean methylation levels. This indicates that we might wrongly detect or fail to detect epigenetic mutations from $450 \mathrm{k}$ chip data. In general, pyrosequencing data were more stable and changes in methylation levels were smoother than that from 450k array (Fig. 5). For example, in cg05270750 measured by the $450 \mathrm{k}$ array (Fig. 5e), one participant was identified to have epigenetic mutations in the first three measures, but the methylation level turned back to normal status in the last two measures. However, pyrosequencing data showed the methylation levels of the five measures from this individual were consistently defined as epigenetic mutations.

\section{Functional validation of epigenetic mutations in cancer tissues}

To further verify the overabundance of epigenetic mutations in cancer tissues, we picked a gene PR/SET domain
7 (PRDM7) which was the only gene related to CpGs tested in pyrosequencing (cg05270750), and analyzed DNA methylation and gene expression data of the gene in tumor tissues and normal adjacent tissues using The Cancer Genome Atlas (TCGA) [16] data downloaded from Wanderer [17]. We selected the four most common cancer types in both sexes combined: lung cancer, breast cancer, colorectal cancer, and prostate cancer [18]. The total numbers of tumor and normal adjacent samples were 2, 209 and 261 respectively, all cancer types combined. On average, the expression levels of PRDM7 were higher in tumor tissues than normal adjacent tissues in all cancer types, but the difference was only statistically significant for lung cancer ( $p=1.83 \mathrm{e}-09$, Additional file 1: Table S3). For DNA methylation data, the tumor tissues had significantly lower methylation levels than normal adjacent tissues in the gene body (Fig. 6a). However, for CpGs in the PRDM7 promoter (from cg06295223 to cg26935333), there was no significant difference between the mean

Table 4 Results from $t$ tests comparing methylation levels in samples with epigenetic mutations to normal samples using data from the $450 \mathrm{k}$ array and pyrosequencing

\begin{tabular}{|c|c|c|c|c|}
\hline \multirow[t]{2}{*}{ Data } & \multicolumn{2}{|c|}{ Number of samples classified by $450 \mathrm{k}$ results } & \multirow{2}{*}{$\begin{array}{l}\text { Mean } \\
\text { difference } \\
\text { (methylation } \\
\text { level, \%) }\end{array}$} & \multirow[t]{2}{*}{$p$ value } \\
\hline & Normal & Mutation & & \\
\hline cg05270750, 450 k-chip & \multirow[t]{2}{*}{81} & \multirow[t]{2}{*}{12} & 13.39 & $4.34 \mathrm{e}-6$ \\
\hline cg05270750, Pyroseq & & & 10.79 & $2.01 e-3$ \\
\hline cg17338133, 450 k-chip & \multirow[t]{2}{*}{76} & \multirow[t]{2}{*}{17} & 13.11 & $6.39 e-8$ \\
\hline cg17338133, Pyroseq & & & 9.35 & 0.02 \\
\hline cg25351353, 450 k-chip & \multirow[t]{2}{*}{67} & \multirow[t]{2}{*}{26} & 14.58 & $7.93 e-17$ \\
\hline cg25351353, Pyroseq & & & 12.70 & $9.20 \mathrm{e}-8$ \\
\hline cg05124918, 450 k-chip & \multirow[t]{2}{*}{63} & \multirow[t]{2}{*}{30} & 21.87 & $3.22 \mathrm{e}-20$ \\
\hline cg05124918, Pyroseq & & & 11.08 & $3.76 e-07$ \\
\hline
\end{tabular}



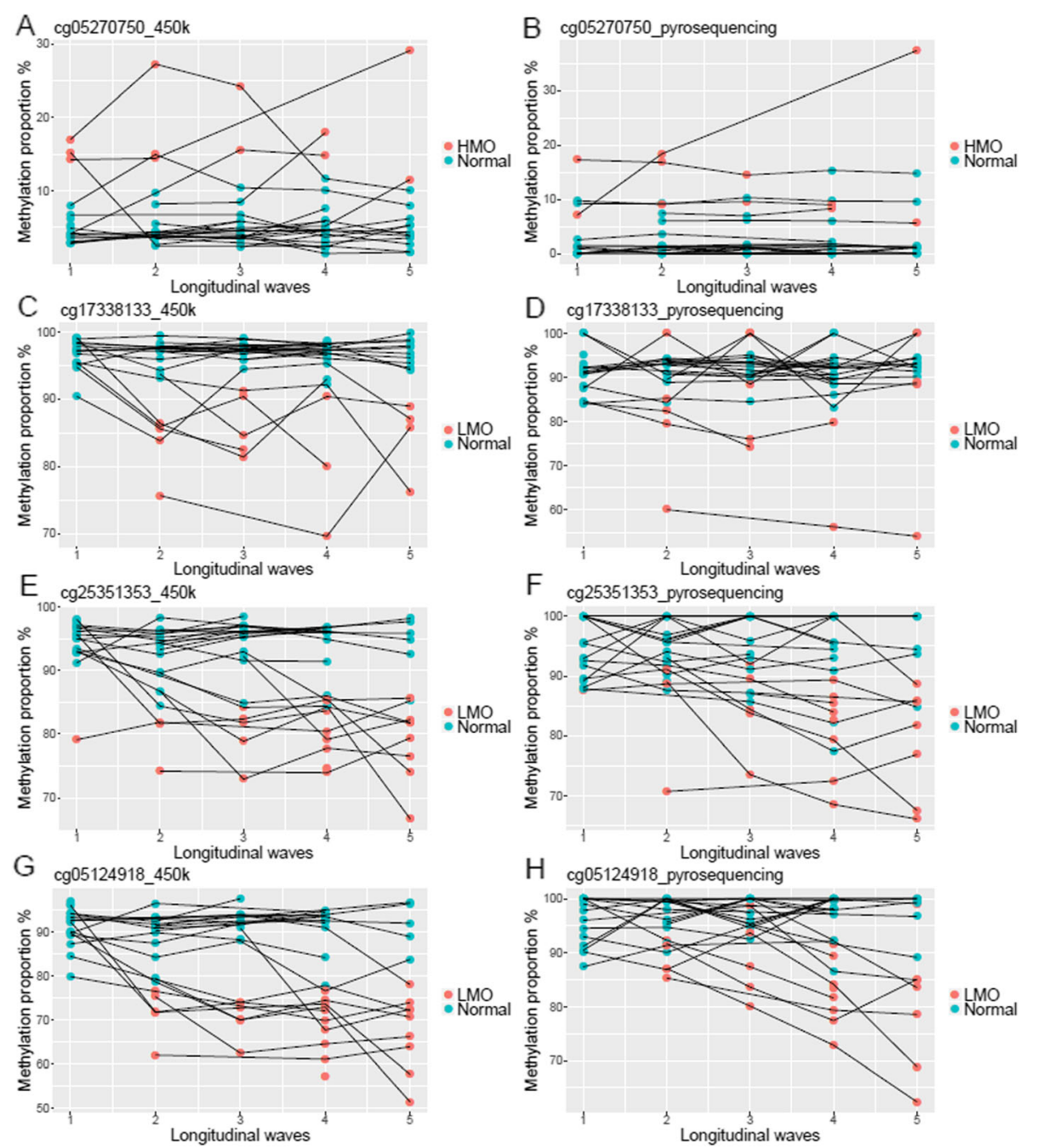

Fig. 5 The longitudinal change of four CpGs in 93 samples from 26 individuals measured by 450k array (left panel) and pyrosequencing (Pyroseq, right panel) techniques. Methylation levels of a cg05270750 from $450 \mathrm{k}$-chip, b cg05270750 from Pyroseq, c cg17338133 from $450 \mathrm{k}$-chip, d cg17338133 from Pyroseq, e cg25351353 from 450 k-chip, f cg25351353 from Pyroseq, g cg05124918 from 450-chip, and $\mathbf{h}$ cg05124918 from Pyroseq. Samples are shown as points colored by their mutation status defined by the 450k data and lines links longitudinal samples collected in the same individual

methylation levels of cancer and normal adjacent tissues (Fig. 6a). To quantify and compare epigenetic mutations in both tissues, we used the distribution of normal adjacent samples to determine epigenetic mutation cutoffs. By calculating the number of epigenetic mutations in tissue samples, tumor tissues had higher proportions of epigenetic mutations in the gene body, while epigenetic mutations were not observed in normal adjacent tissues. In the gene promoter, tumor and normal adjacent tissues had similar and relatively low proportions of epigenetic mutations (Fig. 6b).

\section{Sensitivity analysis}

To test how much age may influence the definition of epigenetic mutations, we identified epigenetic mutations in each of the two age groups (age below or above 75) instead of applying the same cutoffs defined by the first observation. In general, the numbers of epigenetic mutations identified from both methods were highly correlated $(r=0.98)$ across samples. Also, the outlier cutoffs identified from the two age groups were also highly correlated across CpGs ( $r=0.97$ for HMOs and $r=0.98$ for LMOs). As expected, the effect size of age on epigenetic mutation identified from the age groups became smaller $(\beta=4.04 \mathrm{e}-3, p=4.40 \mathrm{e}-4)$ while the effects of other covariates remained similar.

\section{Discussion}

In this study, we analyzed age-related accumulation of epigenetic mutations from a longitudinal perspective in 


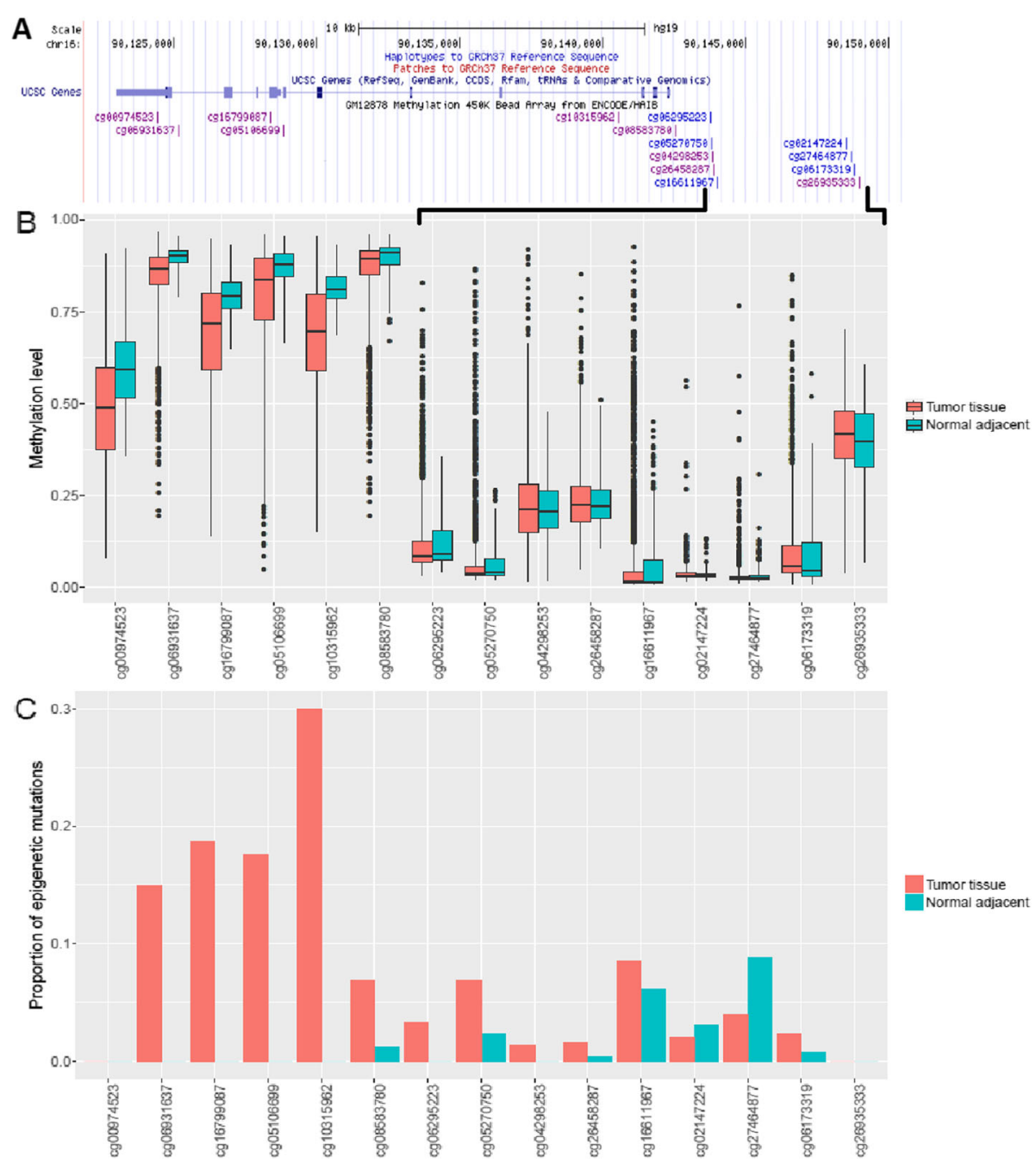

Fig. 6 Comparing the DNA methylation and epigenetic mutation patterns of gene PRDM7 between tumor and normal adjacent tissues. Data were downloaded from TCGA through Wanderer. The cancer types included lung cancer, breast cancer, colorectal cancer, and prostate cancer. a The location of CpGs related to gene PRDM7 in UCSC genome browser. $\mathbf{b}$ The methylation levels of CpGs in gene PRDM7. Tumor and normal adjacent tissues had similar methylation levels in the gene promoter, while the methylation levels of tumor tissues in the gene body were significantly lower than normal adjacent tissues. c The proportion of epigenetic mutations in tumor and normal adjacent tissues. Tumor tissues had higher proportions of epigenetic mutations in the gene body, while both tumor and normal adjacent tissues had similar but low proportion of epigenetic mutations in the gene promoter

old Swedish twins. Apart from being exponentially associated with age, epigenetic mutations were also associated with sex, CD19+ B cell count, genetic background, cancer incidence, and technical factors. We further analyzed frequent HMOs and LMOs separately, and identified similar numbers of $\mathrm{CpGs}$ with HMOs and LMOs but significantly more CpGs with frequent HMOs than frequent LMOs, which was consistent with a previous publication [11]. We also found that biological factors, including $\mathrm{B}$ cell compositions and genetic factors, were more strongly associated with frequent HMOs than LMOs, while frequent LMOs were more influenced by technical factors. Moreover, cancer diagnosis was significantly associated with the increase of epigenetic mutations, especially among frequent HMOs, while the same was not true for LMOs.

Emerging evidence indicate that epigenetic mutations could be related to cancer $[6,11]$, as epigenetic mutations may cause abnormal gene expression, which could contribute to the development of cancer. On the other hand, mutated DNA sequences and abnormal epigenetic regulation in tumor cells may in turn cause more epigenetic mutations. In this study, we found that the number of epigenetic mutations was significantly higher in samples of individuals who were diagnosed with cancer by the end of follow-up. Therefore, we conclude that the number of epigenetic mutations may accumulate long before the diagnosis of cancer. The survival analysis 
further showed that a higher number of frequent HMOs could be a risk factor for cancer incidence. These results support a previous finding where the number of epigenetic mutations were higher in tumor tissues than in normal tissues [12]. Follow-up studies with more participants are needed to better establish the possible relationship between epigenetic mutations and cancer.

In this study, DNA methylation data were corrected for cellular compositions predicted by the Houseman method [19], yet imputed CD19+ B cell count was significantly associated with epigenetic mutations, but not other cell types. A possible explanation could be that B cells have a unique methylation pattern compared to other lymphocytes [20]. Also, B cell composition was still a strong factor for frequent HMOs but the effect became very week for frequent LMOs, probably because cell specific CpGs are enriched in promoter regions [20] where HMOs are mostly found.

When studying functional annotations associated with the epigenetic mutations, we found that the location and regulatory features were different for frequent $\mathrm{HMOs}$ and LMOs. The observed enrichment of HMOs in CpG islands and promoter regions indicated that HMOs were more related to biological function than LMOs, which is in line with the fact that technical bias was significant in LMOs.

The concept of epigenetic mutations should be discussed in relation to methylation variability, as they both describe methylation divergence between individuals. However, epigenetic mutations refer to more extreme methylation levels carried by a small number of individuals, while methylation variability is considered to be a population pattern. In contrast to traditional association studies on methylation levels, where CpGs of higher variances are more likely to have statistical power, CpGs of high variances could have too large inter quartile ranges to be identified as epigenetic mutations by definition. Therefore, the identified frequent epigenetic mutations were different from the age-associated $\mathrm{CpGs}$ or agevaried CpGs reported prior to this study using the same data $[3,5]$, and thus may contribute to the aging processes by other ways than through the epigenetic drift.

Even after excluding cis-meQTL CpGs, a small genetic effect captured by the first genetic PC was associated with epigenetic mutations, especially in frequent HMOs. To further explore how genetic background and age affected the accumulation of epigenetic mutations, we studied the number of shared epigenetic mutations between twins over time. Here we did not simply exclude cis-meQTL CpGs, but considered them as epigenetic mutations caused by genetic variants inherited at birth. For all CpGs and non-meQTL CpGs, we observed both age and genetic effect associated with the number of shared epigenetic mutations within the twin pair. To isolate the genetic effect, we specifically analyzed cismeQTL CpGs and found that in this selection, the number of shared epigenetic mutations did not change with age. This result was consistent with a previous paper showing that meQTL-CpG associations are stable over time [21]. Additionally, we failed to detect an interaction between genetic factors and age, indicating that the increase of epigenetic mutations with age was not dependent on the genetic background. Therefore, the remaining genetic effect observed after removing cismeQTL CpGs was probably due to trans-meQTLs or unidentified cis-meQTLs. In conclusion, the age effect on the accumulation of epigenetic mutations is independent of genetic background. However, we might not have enough statistical power to detect a significant agegenetic interaction on shared epigenetic mutations, since the age effect estimated for MZ twins was larger than for DZ twins. Moreover, due to the limit of the age range in this study (48 to 98 years), we could not exclude the possibility of genetic-associated development of epigenetic mutations in early ages, which remains to be examined by future studies.

Technical artifacts and poor sample quality could lead to erroneous measures that interfere with identifying true biological methylation outliers. Although sample quality control based on detection $p$ value was applied in the pre-processing pipe-line of the methylation data, it was still found to strongly influence the identification of the epigenetic mutations. Although the technical effect was strong and hard to avoid, the effect of age on epigenetic mutations was not biased as we randomized samples on microarrays. Another important technical artifact is the batch effect from different arrays, but we adjust for batches both in data pre-processing and as a random effect in the mixed effect model. Hence, despite the confounding issues from different technical biases when analyzing methylation outliers, the underlying biological phenomenon of increasing number of epigenetic mutations with age still holds.

Validation of the epigenetic mutations identified in $450 \mathrm{k}$ data was done by pyrosequencing, which also detected aberrant methylation levels proving that they were true biological outliers and not simply technical errors. However, some samples showed very different results between the two methods suggesting measurement errors existed. When comparing results from the two methods, pyrosequencing data were more stable and better indicated that epigenetic mutations were persistent over time, which supported the accumulation of epigenetic mutations as a factor of aging.

The HMO site cg05270750 validated by pyrosequencing is located in the promoter region of the gene PRDM7, which encodes a Histone-Lysine Trimethyltransferase involved in histone modification. To further 
explore the potential consequence of epigenetic mutations, we analyzed DNA methylation and gene expression of gene PRDM7 in data on tumor and normal adjacent tissues from TCGA. The expression of PDM7 in normal adjacent tissues was very low, as previously seen [22]. Nevertheless, we observed higher expression of PRDM7 in tumor tissues, especially in lung cancers, suggesting the abnormal expression of PRDM7 could be related to the dysregulation of histone modification in tumor. On the other hand, we observed similar proportions of epigenetic mutations between tumor and normal adjacent tissues in the gene promoter, but more epigenetic mutations in the gene body for tumor tissues. Since normal adjacent tissue can be regarded as an intermediate state between healthy and tumor tissues, it is suggested that, in the process of cancer development, epigenetic mutations were likely to first accumulate in gene promoters and then spread to the whole epigenome.

\section{Conclusions}

In summary, using longitudinal DNA methylation data, we showed that the accumulation of epigenetic mutations is exponentially associated with age in old adults, and once mutations are established, they are stable over time. Furthermore, epigenetic mutations are enriched in important regulatory sites, e.g., promoter regions of genes involved in histone modification processes, which could potentially be an explanation to why people who develop cancer have more epigenetic mutations than others do. In addition, we showed that the burden of accumulation associated with the human aging process is unlikely to be driven by underlying genetic background. Hence, accumulation of epigenetic mutations is an underexplored area in the field of aging and warrants further studies to enhance our understanding of this phenomenon.

\section{Methods}

\section{Study population}

Twins as participants in this study were enrolled in the SATSA longitudinal cohort study [23]. After quality control, a total of 994 blood samples obtained from 375 individuals in five longitudinal waves (1992-2012) were used in the analyses. The 375 participants had a mean age of 68.9 years $(S D=9.7)$ at their first measurement, and 223 (59.5\%) were women. Of the 375 participants, 197 contributed samples in three or more waves. Phenotype data were collected through comprehensive questionnaires and physical testing at each sampling wave. Phenotypes used in this study include chronological age, sex, zygosity, smoking status, and cancer diagnosis.

\section{DNA methylation data}

DNA methylation data were obtained from DNA extracted from whole blood samples measured by Infinium HumanMethylation450 BeadChips. In total, 485,512 CpG sites were measured for each sample. The quality control and preprocessing methods of the methylation data were described in a previous study [3]. Samples from individuals lacking genetic data were removed, retaining a total of 994 samples for analyses. Blood cellular compositions were estimated by the Houseman method [19] using a reference panel [20]. The methylation data were adjusted by cellular compositions using a linear regression before the analyses. Additionally, batch effects, which were detected as slides on the 450k chip, were adjusted using the Combat method from the sva package [24].

\section{Genotype data and imputation}

Genetic data were measured by Infinium PsychArray (Illumina Inc., San Diego, CA, USA) with 588,454 SNPs detected for every individual. After quality control, data were imputed to the 1000 Genomes Project phase 1 version 3 reference [25] using IMPUTE2 version 2.3.2 [26, 27] with default parameters. The first 10 PCs were calculated based on a linkage disequilibrium pruned set of directly genotyped autosomal SNPs.

\section{Identifying epigenetic mutations}

The definition of an epigenetic mutation was consistent with Gentilini et al [7]. For each CpG, the quartiles of methylation levels were calculated for every CpG using the first observation available from each individual, and were calculated separately for men and women to avoid the sex effect on methylation levels. Samples having methylation levels three times the inter quartile range higher than the third quartile or lower than the first quartile were identified as mutated outliers. Methylation levels were presented in beta-values, which indicate the methylation proportions. CpGs associated with cismeQTLs (<1 Mbps) were removed from further epigenetic mutation analyses. For the rest of the CpGs, outlier samples were identified as epigenetic mutations, and the total number of epigenetic mutations was counted for every sample. Identified epigenetic mutations were classified into HMOs and LMOs according to whether they exceed the upper or lower boundary of normal methylation levels (defined as 3 times IQR higher than the third quantile or lower than the first quantile).

\section{Statistical analysis}

A mixed effect model was fitted to measure the association of the number of epigenetic mutations on age and other factors (Eq. 1). A log-10 transformation was applied to the number of epigenetic mutations to form a 
distribution closer to a normal distribution. For each sample, the log10-transformed number of CpGs with detection $p$ values over 0.01 was used to indicate the sample quality. In the formula, $i, j$, and $k$ denote individual, slide batch, and waves; $\beta_{0}, \beta_{1}, \beta_{2}, \beta_{3}, \beta_{4}, \beta_{5}$, and $\beta_{6}$ denote fixed intercepts, fixed coefficient of age, sex, CD19 $\mathrm{B}$ cell composition, first genetic principal component, detection $p$ value, and whether the individual developed cancer; $\mathrm{u} 0$, u1, and $\varepsilon$ denotes random intercept of individual, slide batch, and random error.

$$
\begin{aligned}
\text { Mut }_{i, j, k}= & \beta_{0}+\beta_{1} \text { Age }_{i, j, k}+\beta_{2} \operatorname{Sex}_{i}+\beta_{3} \text { Beell }_{i, j, k} \\
& +\beta_{4} \mathrm{PC1}_{i}+\beta_{5} \operatorname{Dpval}_{i, j, k}+\beta_{6} \text { Cancer }_{i} \\
& +u_{0 i}+u_{1 j}+\varepsilon_{i, j, k}
\end{aligned}
$$

The survival analysis of cancer diagnosis and epigenetic mutations was performed using a Cox model. The model included sex, current smoking as baseline exposure, number of epigenetic mutations as a time-varying covariate, and attained age as the time scale. The model was further adjusted for twin pair and batch effect using robust standard error.

In twin analysis, a mixed effect model was used to study the number of exact same epigenetic mutations between paired twins measured at the same time in association with age, sex, and twin zygosity (Eq. 2),

$$
\begin{aligned}
\log _{10} N_{i, j}= & \beta_{0}+\beta_{1} \mathrm{Age}_{i, j}+\beta_{2} \operatorname{Sex}_{i}+\beta_{3} \mathrm{Zyg}_{i} \\
& +\beta_{4} \mathrm{Zyg}_{i} \times \mathrm{Age}_{i, j}+u_{0 i}+\varepsilon_{i, j}
\end{aligned}
$$

where $i$ and $j$ denote individual and longitudinal measure; $\beta_{0}, \beta_{1}, \beta_{2}, \beta_{3}, \beta_{4}$ denote fixed intercept, fixed coefficient of age, sex, zygosity, and zygosity-age interaction; $u_{0 i}$, and $\varepsilon$ denote random intercept of individual and random error.

All statistical analyses were performed in R version 3.4.3.

\section{Pyrosequencing}

In total, 93 samples from 26 individuals were measured by pyrosequencing to validate epigenetic mutations in 4 CpGs (cg05270750, cg17338133, cg25351353, cg05124918). The samples were selected to present 4 to 5 longitudinal measures for every individual. The selection of CpGs was based on their primer quality, and having large numbers of mutated samples. The primers of the four CpGs were designed using the software PyroMark Assay Design by QIAGEN. DNA samples were converted by bisulfite reaction performed on EZ-96 DNA Methylation-Gold ${ }^{\mathrm{mm}}$ MagPrep kit provided by ZYMO RESEARCH CORP. Converted samples were randomized in a 96-well plate and sequenced for each CpG on PyroMark Q96 ID provided by QIAGEN. The raw data were processed in PyroMark Q24 Software v2.5.8 by QIAGEN.

\section{Supplementary information}

Supplementary information accompanies this paper at https://doi.org/10. 1186/s13148-019-0788-9.

Additional file 1: Figure S1. The distribution of number of epigenetic mutations. A) The number of epigenetic mutations showed a rightskewed distribution. B) After log-transformation, the distribution is close to normal distribution. Figure S2. The examples of epigenetic mutations in two directions. The distribution of methylation levels of A) cg02018277 (higily methylated outlier) and B) cg15057747 (lowly methylation outlier). The methylation levels of epigenetic mutations were greatly different to normal samples, exceeding three times of inter quantile range of the first or third quantile. The samples in red had epigenetic mutations and their methylation levels greatly differed to normal samples in blue. Figure S3. The number of epigenetic mutations shared between twins in association with age and twin zygosity. The number of shared epigenetic mutations significantly increased with age for A) all CpGs and B) non-meQTL CpGs. In both cases, monozygotic twins (MZ) shared more epigenetic mutations than dizygotic twins (DZ), indicating genetic effect. C) But for meQTL CpGs, epigenetic mutations shared by twins was not associated with age which suggested that meQTLs were stable over time. None of the three regressions showed age-zygosity interaction, indicating that genetic does not influence the rate of age-associated increase of epigenetic mutations. Table S1. The association of epigenetic mutations with age and covariates from mixed model using individuals with four or more measures. Table S2. The survival analysis of epigenetic mutations in association with cancer incidence using a Cox proportional hazard model. For all epigenetic mutations, frequent HMOs and frequent LMOs, the hazard ratios represent the ratio of an increase of 10 epigenetic mutations. Table S3. The mean expression levels of gene PRDM7 in tumor and normal adjacent tissues for different cancer types. A t-test was used to compare the mean expression levels in tumor and normal adjacent tissues. The gene expression data were downloaded from TCGA through Wanderer.

\section{Abbreviations}

aDMS: Age-associated differentially methylated site; CpG: 5'-Cytosinephosphate-Guanine-3'; CTCF: CCCTC-Binding factor; HMO: High methylation outliers; IQR: Interquartile ranges; LMO: Low methylation outliers; Mbps: Mega base pairs; meQTL: Methylation quantitative loci; PC: Principal component; PRDM7: PR/SET domain 7; SATSA: Swedish Adoption/Twin Study of Aging; SNP: Single nucleotide polymorphism; TCGA: The Cancer Genome Atlas

\section{Acknowledgements}

The results published here are in part based upon data generated by the TCGA Research Network: https://www.cancer.gov/tcga.

\section{Authors' contributions}

$\mathrm{SH}, \mathrm{NP}$, and YW conceived and designed this study. YW performed data processing and statistical analysis and drafted the manuscript. YW and MA conducted pyrosequencing for validation. SH, ÅH, RK, JJ, IK, and MA contributed to the manuscript writing. All authors read and approved the final manuscript.

\section{Funding}

This study was supported by NIH grants R01 [AG04563, AG10175, AG028555], the MacArthur Foundation Research Network on Successful Aging, the European Union's Horizon 2020 research and innovation program [No. 634821], the Swedish Council for Working Life and Social Research (FAS/FORTE) [97:0147:1B, 2009-0795, 2013-2292], the Swedish Research Council [825-2007-7460, 825-20096141, 521-2013-8689, 2015-03255, 2015-06796], the Karolinska Institutet delfinansiering (KID) grant for doctoral students (YW), the KI Foundation, the Strategic Research Area in Epidemiology at Karolinska Institutet, Erik Rönnbergs donation for scientific studies in aging and age-related diseases, and King Gustaf V:s and Queen Victorias Freemason Foundation. Open access funding provided by Karolinska Institute. 


\section{Availability of data and materials}

The datasets generated and analyzed during the current study are available in Array Express database of EMBL-EBL (www.ebi.ac.uk/arrayexpress) under accession number E-MTAB-7309.

\section{Ethics approval and consent to participate}

All participants in SATSA have provided written informed consents. This study was approved by the ethics committee at Karolinska Institutet with Dnr 2015/1729-31/5.

\section{Competing interests}

The authors declare that they have no competing interests.

\section{Author details}

${ }^{1}$ Department of Medical Epidemiology and Biostatistics, Karolinska Institutet, Nobels väg 12A, 17177 Stockholm, Sweden. ${ }^{2}$ Rheumatology Unit, Department of Medicine Solna, Karolinska Institutet, Stockholm, Sweden. ${ }^{3}$ Pfizer Worldwide Research and Development, Stockholm, Sweden. ${ }^{4}$ Astrid Lindgren Children's Hospital, Karolinska University Hospital, Stockholm, Sweden. ${ }^{5}$ Institute of Gerontology and Aging Research Network - Jönköping (ARN-J), School of Health and Welfare, Jönköping University, Jönköping, Sweden. ${ }^{6}$ Department of Clinical Neuroscience, Centrum for Molecular Medicine, Karolinska Institutet, Stockholm, Sweden.

Received: 13 May 2019 Accepted: 25 November 2019

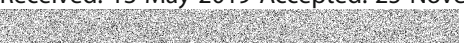

\section{References}

1. López-Otín C, Blasco MA, Partridge L, Serrano M, Kroemer G. The hallmarks of aging. Cell. 2013;153(6):1194-217.

2. Bell JT, Tsai P-C, Yang T-P, Pidsley R, Nisbet J, Glass D, et al. Epigenome-wide scans identify differentially methylated regions for age and age-related phenotypes in a healthy ageing population. PLoS Genet. 2012;8(4): e1002629.

3. Wang Y, Karlsson R, Lampa E, Zhang Q, Hedman ÅK, Almgren M, et al. Epigenetic influences on aging: a longitudinal genome-wide methylation study in old Swedish twins. Epigenetics. 2018;13(9):975-87.

4. Fraga MF, Ballestar E, Paz MF, Ropero S, Setien F, Ballestar ML, et al. Epigenetic differences arise during the lifetime of monozygotic twins. PNAS. 2005:102(30):10604-9.

5. Wang Y, Pedersen NL, Hägg S. Implementing a method for studying longitudinal DNA methylation variability in association with age. Epigenetics. 2018;13(8):866-74.

6. Feinberg AP, Koldobskiy MA, Göndör A. Epigenetic modulators, modifiers and mediators in cancer aetiology and progression. Nat Rev Genet. 2016; 17(5):284-99.

7. Gentilini D, Garagnani P, Pisoni S, Bacalini MG, Calzari L, Mari D, et al. Stochastic epigenetic mutations (DNA methylation) increase exponentially in human aging and correlate with $X$ chromosome inactivation skewing in females. Aging (Albany NY). 2015;7(8):568-78.

8. Teschendorff AE, Gao Y, Jones A, Ruebner M, Beckmann MW, Wachter DL, et al. DNA methylation outliers in normal breast tissue identify field defects that are enriched in cancer. Nat Commun. 2016;7:10478.

9. Teschendorff AE, Jones A, Widschwendter M. Stochastic epigenetic outliers can define field defects in cancer. BMC Bioinformatics. 2016;17(1):178.

10. Ghosh J, Mainigi M, Coutifaris C, Sapienza C. Outlier DNA methylation levels as an indicator of environmental exposure and risk of undesirable birth outcome. Hum Mol Genet. 2016:25(1):123-9.

11. Ghosh J, Schultz B, Coutifaris C, Sapienza C. Highly variant DNA methylation in normal tissues identifies a distinct subclass of cancer patients. Adv Cancer Res. 2019;142:1-22.

12. Gentilini D, Scala S, Gaudenzi G, Garagnani P, Capri M, Cescon M, et al. Epigenome-wide association study in hepatocellular carcinoma: identification of stochastic epigenetic mutations through an innovative statistical approach. Oncotarget. 2017:8(26):41890-902

13. Magnusson PKE, Almqvist C, Rahman I, Ganna A, Viktorin A, Walum H, et al. The Swedish Twin Registry: establishment of a biobank and other recent developments. Twin Res Hum Genet. 2013;16(1):317-29.

14. Zerbino DR, Wilder SP, Johnson N, Juettemann T, Flicek PR. The ensembl regulatory build. Genome Biol. 2015;16:56.
15. Huang DW, Sherman BT, Lempicki RA. Systematic and integrative analysis of large gene lists using DAVID bioinformatics resources. Nat Protoc. 2009:4(1): 44-57.

16. The Cancer Genome Atlas Home Page. The Cancer Genome Atlas - National Cancer Institute. 2011. Available from: https://cancergenome.nih.gov/. [cited 2019 Feb 6].

17. Díez-Villanueva A, Mallona I, Peinado MA. Wanderer, an interactive viewer to explore DNA methylation and gene expression data in human cancer. Epigenetics Chromatin. 2015;8(1):22.

18. BW S, CP W. World Cancer Report 2014. Available from: http://publications. iarc.fr/Non-Series-Publications/World-Cancer-Reports/World-Cancer-Report-2 014. [cited 2019 Mar 1].

19. Houseman EA, Accomando WP, Koestler DC, Christensen BC, Marsit CJ, Nelson $\mathrm{HH}$, et al. DNA methylation arrays as surrogate measures of cell mixture distribution. BMC Bioinformatics. 2012;13(1):86.

20. Reinius LE, Acevedo N, Joerink M, Pershagen G, Dahlén S-E, Greco D, et al. Differential DNA methylation in purified human blood cells: implications for cell lineage and studies on disease susceptibility. PLoS ONE. 2012;7(7): e41361.

21. Gaunt TR, Shihab HA, Hemani G, Min JL, Woodward G, Lyttleton O, et al. Systematic identification of genetic influences on methylation across the human life course. Genome Biol. 2016:17(1):61.

22. GTEx Portal [Internet]. Available from: https://gtexportal.org/home/gene/ PRDM7. [cited 2019 Mar 1].

23. Finkel D, Pedersen NL. Processing speed and longitudinal trajectories of change for cognitive abilities: the Swedish Adoption/Twin Study of Aging Aging Neuropsychol Cogn. 2004;11(2-3):325-45.

24. Leek JT, Johnson WE, Parker HS, Jaffe AE, Storey JD. The sva package for removing batch effects and other unwanted variation in high-throughput experiments. Bioinformatics. 2012;28(6):882-3.

25. The 1000 Genomes Project Consortium, Auton A, Abecasis GR, Altshuler DM, Durbin RM, Abecasis GR, et al. A global reference for human genetic variation. Nature. 2015:526:68

26. Howie BN, Donnelly P, Marchini J. A flexible and accurate genotype imputation method for the next generation of genome-wide association studies. PLoS Genet. 2009;5(6):e1000529.

27. Howie B, Marchini J, Stephens M. Genotype imputation with thousands of genomes. G3 (Bethesda). 2011;1(6):457-70.

\section{Publisher's Note}

Springer Nature remains neutral with regard to jurisdictional claims in published maps and institutional affiliations.

Ready to submit your research? Choose BMC and benefit from:

- fast, convenient online submission

- thorough peer review by experienced researchers in your field

- rapid publication on acceptance

- support for research data, including large and complex data types

- gold Open Access which fosters wider collaboration and increased citations

- maximum visibility for your research: over $100 \mathrm{M}$ website views per year

At BMC, research is always in progress.

Learn more biomedcentral.com/submissions 